\title{
Defects in GaN Pyramids Grown on Si(111) Substrates by Selective Lateral Overgrowth
}

\author{
ZHIGANG MAO*, STUART MCKERNAN*, C. BARRY CARTER*, WEI YANG** and \\ SCOTT A. McPHERSON** \\ *Department of Chemical Engineering and Materials Science, University of Minnesota, \\ MN55455-0132, U.S.A. \\ **Honeywell Technology Center, 12001 State Hwy. 55, Plymouth, MN 55441, U. S. A
}

\section{ABSTRACT}

Selective lateral growth of $\mathrm{GaN}$ is a promising technique for producing high quality material for microelectronic and optoelectronic devices. Single-crystal GaN/AIN layers have been grown on $\mathrm{Si}(111)$ substrates and subsequently used as the seeding layer for selective lateral overgrowth. $\mathrm{GaN}$ pyramids are formed above holes patterned in a $\mathrm{Si}_{3} \mathrm{~N}_{4}$ mask. Transmission electron microscopy (TEM, which also denotes the microscope) of these structures shows that the GaN pyramid, GaN seed layer, and AlN buffer layer in the samples have the following epitactic relationship with respect to the Si substrate: $[11 \overline{2} 0]_{\mathrm{GaN}}\left\|[11 \overline{20}]_{\mathrm{AlN}}\right\|[\overline{1} 10]_{\mathrm{Si}}$ and $(0001)_{\mathrm{GaN}} \|$ $(0001)_{\mathrm{AlN}} \|(111)_{\mathrm{Si}}$. In the core of the pyramid (at or above the seed windows), dislocations thread through the pyramid perpendicular to the interface plane with very high density. Some of these threading dislocations, which originate from the GaN/AIN seed layer, form $90^{\circ}$ bends and half loops at the edge of the pyramid core. In the lateral growth part of the GaN pyramid, the dislocation density is relatively low. The majority of dislocations thread through the pyramid parallel to the interface plane. Planar defects, usually parallel to the interface plane, were observed near the interface. The defect density decreases with the distance away from the interface, so that the top several microns of material maybe completely defect free. The mechanism of the growth of GaN pyramids is discussed and related to this defect structure.

\section{INTRODUCTION}

III-V nitrides have been extensively investigated for applications in microelectronic and optoelectronic devices because of their wide direct bandgap and good thermal, chemical, and mechanical stability. Selective growth is an important techniques in the fabrication of field effect transistors (FETs) [1] and semiconductor micro-device structures such as quantum wires and dots [2]. Selective growth can also be used to examine the growth mechanisms and the selective epitaxy of $\mathrm{GaN}$.

Selective lateral growth of $\mathrm{GaN}$ on sapphire and $\mathrm{SiC}$ substrates has been reported recently [3]. The growth of single crystal GaN films on these substrates is well documented. Si is the substrate of choice due to its low cost, large size, and the potential for the integration of GaNbased optoelectronic devices with Si-based electronics.

In the present report, the microstructure and the lateral epitaxy mechanism of selectively grown GaN pyramids within windows in $\mathrm{Si}_{3} \mathrm{~N}_{4}$ masks on $\mathrm{Si}(111)$ substrates have been studied by TEM.

\section{EXPERIMENTAL DETAILS}

The GaN growth was conducted in a low-pressure metal-organic chemical-vapor deposition (MOCVD) system with a vertical reactor. A Si(111) wafer was etched in $\mathrm{HF}: \mathrm{H}_{2} \mathrm{O}$, rinsed and dried immediately before loading. The system was evacuated before the pressure was regulated at 10 Torr with a constant $\mathrm{H}_{2}$ flow. The susceptor was inductively heated to $1150{ }^{\circ} \mathrm{C}$ and the $\mathrm{Si}$ wafer was baked at this temperature for 10 minutes under the $\mathrm{H}_{2}$ flow. The AlN buffer layer deposition was initiated by flowing triethylaluminum (TEA) and ammonia $\left(\mathrm{NH}_{3}\right)$ into the reactor. The AlN buffer layer was grown for 20 minutes, resulting in a thickness of $100 \mathrm{~nm}$. A thin GaN layer was then grown with triethylgallium (TEG) for 30 minutes, resulting in a thickness of about $200 \mathrm{~nm}$. The wafer was then deposited with a $100 \mathrm{~nm} \mathrm{Si}_{3} \mathrm{~N}_{4}$ masking layer by plasma-enhanced 
chemical-vapor deposition (PECVD). Arrays of openings of $5 \mu \mathrm{m}$ diameter and $20 \mu \mathrm{m}$ centercenter pitch were formed using photolithography and reactive ion etching (RIE). The wafer was reloaded into the MOCVD system for the lateral overgrowth. The system pressure was 76 Torr. The growth started as soon as the susceptor temperature reached $1050{ }^{\circ} \mathrm{C}$, by flowing ammonia and TEG into the reactor. The total time for the lateral overgrowth was 3 hours.

The cross-sectional specimen was prepared using the usual 'sandwich' techniques: two pieces of the sample with the same orientation were glued face-to-face with epoxy. After mechanical grinding and dimpling of the cross-sectional specimen to a thickness of $\sim 10 \mu \mathrm{m}$, the specimen was further thinned by ion-beam milling to electron transparency. The specimens were then examined in a Philips CM30 TEM operating at $300 \mathrm{kV}$. The analysis of the composition was carried out using an Edax PV9900 XEDS (X-ray energy dispersive spectrum) system attached to the CM30 TEM.

\section{EXPERIMENTAL RESULTS AND DISCUSSION}

Observation by scanning electron microscopy (SEM) indicates that each pyramid has predominantly six $\{10 \overline{1} 1\}$ facets [4]. These facets are identical to those of the pyramids grown on sapphire and SiC substrates [3]. Visible-light microscopy (VLM) of these specimens[4] can reveal the interface between the pyramids and the substrate, and show some fine structures in the center of the pyramids. There is a difference in the contrast between the center part and the lateral part of the pyramids. This difference could be associated with local differences in the defect density and growth mechanism. The height and width of the pyramids are $\sim 15 \mu \mathrm{m}$.

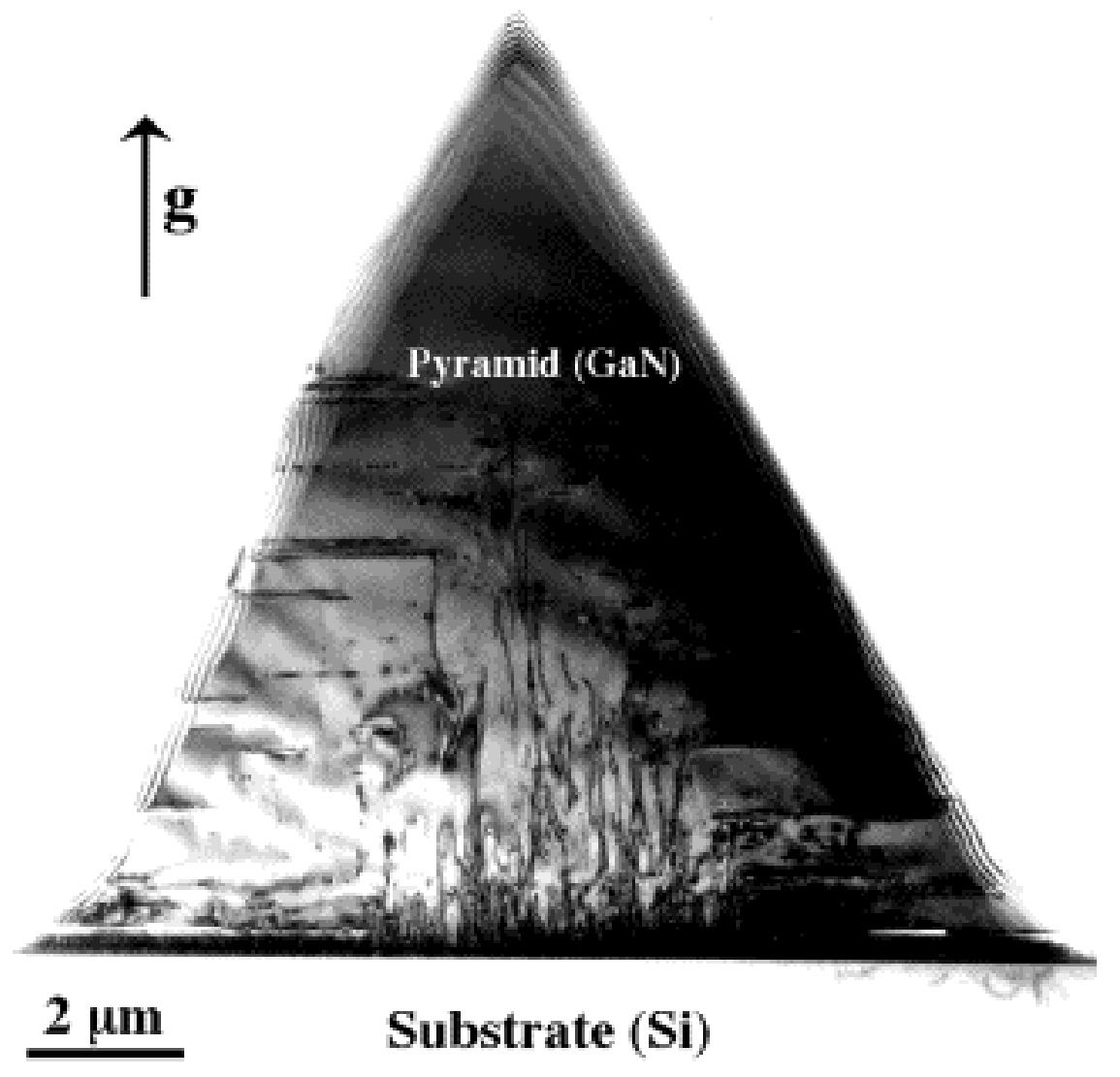

Figure 1 A low-magnification bright-field (BF) image of the cross-sectional specimen showing a pyramid. $\mathbf{g}=0002$. 
Figure 1 is a low-magnification bright-field (BF) image of the cross-sectional specimen. A selectively grown GaN pyramid on $\mathrm{Si}$ substrate was clearly revealed in this image. In the center of the GaN pyramid (at and above the window area), dislocations thread through the pyramid nearly perpendicular to the interface plane and the dislocation density is quite high. In the lateral growth part of the pyramid, the dislocations thread through the pyramid parallel to the interface plane. It is apparent that the density of the defects in the lateral part is lower than the core part (at and above the window area) of the pyramid and decreases with the distance away from the interface. Planar defects, usually parallel to the interface plane, were also observed near the interface. The mask (having a low average atomic number, $Z$ ) is revealed by the bright line between the pyramid and the substrate in the mass-thickness contrast TEM image of the cross-sectional specimen in figure 2. The size of the window in the mask is $\sim 5 \mu \mathrm{m}$. The base of the pyramid is $15 \mu \mathrm{m}$ wide from this image, consistent with the observations of VLM. The GaN pyramids clearly extend laterally well beyond the window. The contrast in the image also confirms that there is a layer between the $\mathrm{Si}_{3} \mathrm{~N}_{4}$ mask and the substrate. At high magnifications, this layer is revealed to contain the expected two layers with thicknesses of $150 \mathrm{~nm}$ and $125 \mathrm{~nm}$, respectively. The thickness of the mask is about $80 \mathrm{~nm}[4]$.

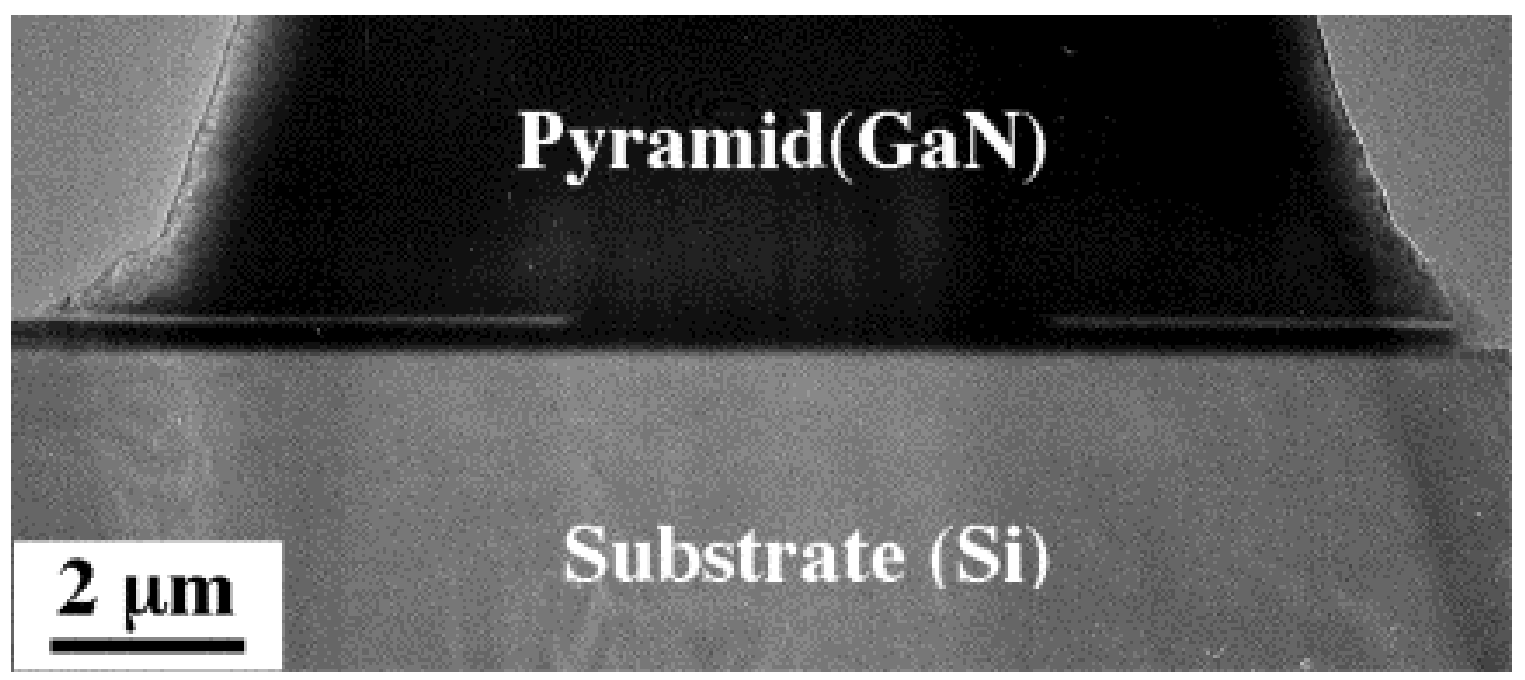

Figure 2 A low-magnification mass-thickness contrast TEM image of the cross-sectional specimen showing the mask.

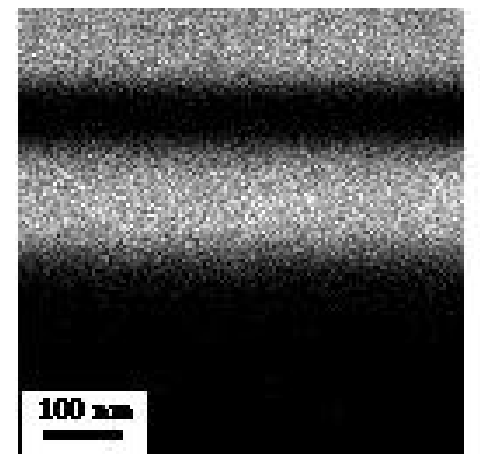

(a)

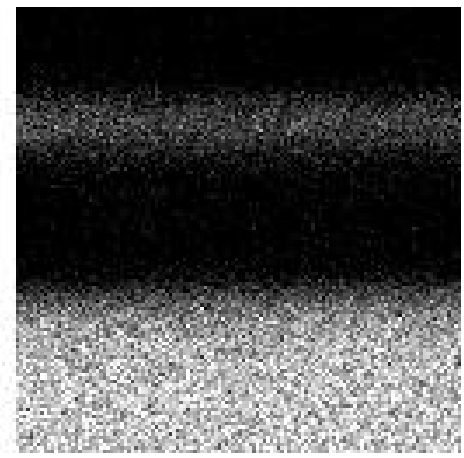

(b)

Figure 3 The X-ray composition map across the interface region. (a) Ga, (b) Si. 
The composition map across the interface is given in Figure 3. The images show the corresponding X-ray composition maps for the elements Ga and Si. They confirm that the pyramid and seed layer are GaN, and the substrate is Si. Selected-area diffraction (SAD) patterns from the interface area, the GaN pyramid, and the substrate confirm that the GaN pyramid, the GaN layer, and the AlN buffer layer are all monocrystalline, with an epitactic relationship with respect to the Si substrate: $[11 \overline{2} 0]_{\mathrm{GaN}}\left\|[11 \overline{2} 0]_{\mathrm{AlN}}\right\|[\overline{110}]_{\mathrm{Si}}$ and $(0001)_{\mathrm{GaN}}\left\|(0001)_{\mathrm{AlN}}\right\|(111)_{\mathrm{Si}}[4]$.

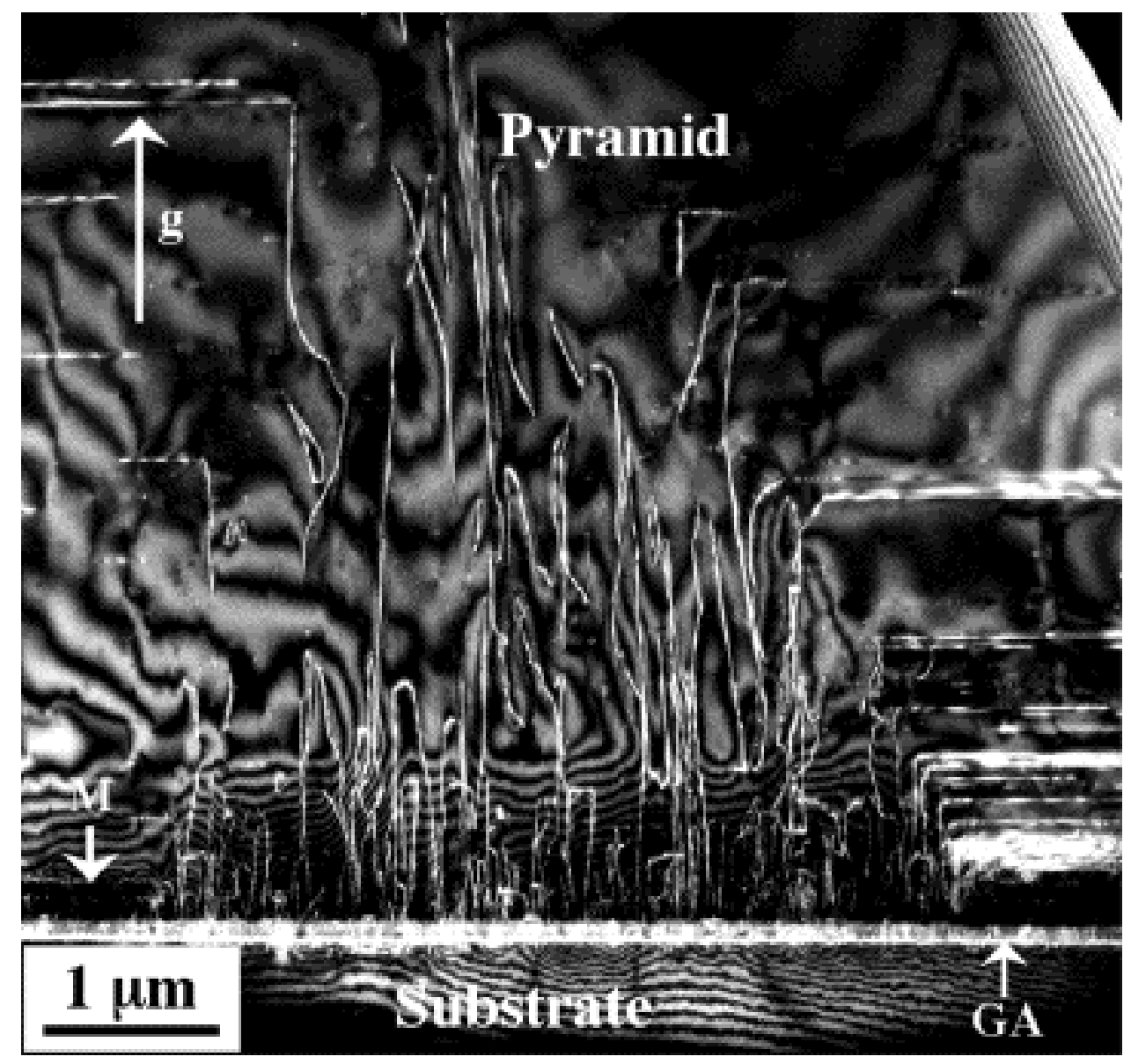

Figure 4 A weak-beam dark-field (WBDF) image taken near the [11 $\overline{2} 0] \mathrm{GaN}$ zone axis with $\mathbf{g} / 4 \mathbf{g}, \mathbf{g}=0002$. In the figure, "M" represents $\mathrm{Si}_{3} \mathrm{~N}_{4}$ mask, "GA" represents the GaN seed layer and the AlN buffer layer.

A representative distribution of the dislocations within the core part in the pyramid is shown in Figure 4. This weak-beam dark-field (WBDF) image is taken near the [1120] GaN zone axis with the $\mathbf{g} / 4 \mathbf{g}$ condition, $\mathbf{g}=0002$. In the figure, "M" represents the $\mathrm{Si}_{3} \mathrm{~N}_{4}$ mask, "GA" represents the GaN seed layer and the AlN buffer layer. Planar defects parallel to the interface plane were clearly revealed near the interface in the lateral growth part of the pyramid. Convergent-beam electron diffraction (CBED) was attempted in order to determine if there was polarity change (inversion) across the boundaries of the planar defects. However, no unambiguous determination could be obtained due to the very high density of the defects in this area.

Further WBDF images were taken from the lateral growth part of the pyramid in order to analyse the dislocation characteristics. Representative images are shown in Figure 5. There are four defects in the WBDF images. These defects (dislocations or dislocation loops) are indicated as $A$, $B, C$, and $D$ in the figures at different conditions. Figure 5 (a) with $\mathbf{g} / 5 \mathbf{g}, \mathbf{g}=1101$, and (b) $\mathbf{g} / 5 \mathbf{g} \mathbf{g}$ $=000 \overline{2}$. In Figures 5(a), the electron beam is nearly parallel to the GaN [11 $\overline{2} 0]$ zone axis and in (b) the $\mathrm{GaN}[0110]$ zone axis. The thickness fringes terminated at the ends of the dislocations can be used to determine the Burgers vector of the dislocations with Ishida's method $[5,6]$. All the 
thickness fringes terminated at the left-hand ends of the dislocations are indicated by arrows. Defects $A, C$, and $D$ are dislocations, while defect $B$ could have an associated dislocation half loop. By using these thickness fringes, together with $\mathbf{g} \cdot \mathbf{b}$ contrast analysis [6], the Burgers vectors of dislocations $A, B, C$, and $D$ were determined to be $1 / 3[\overline{2} 110], 1 / 3[1 \overline{2} 10], 1 / 3[\overline{11} 20]$, and $1 / 3[1 \overline{2} 10]$, respectively. The line directions of dislocations $A, C$, and $D$ were determined to be $1 / 3$ [2110], [ [1010], and $1 / 3[2 \overline{110}$ ], respectively [4], by trace analysis [6]. It is clear that the dislocations observed could be either pure screw or mixed dislocations with a $30^{\circ}$ or $60^{\circ}$ angle between the Burgers vector and the dislocation line.
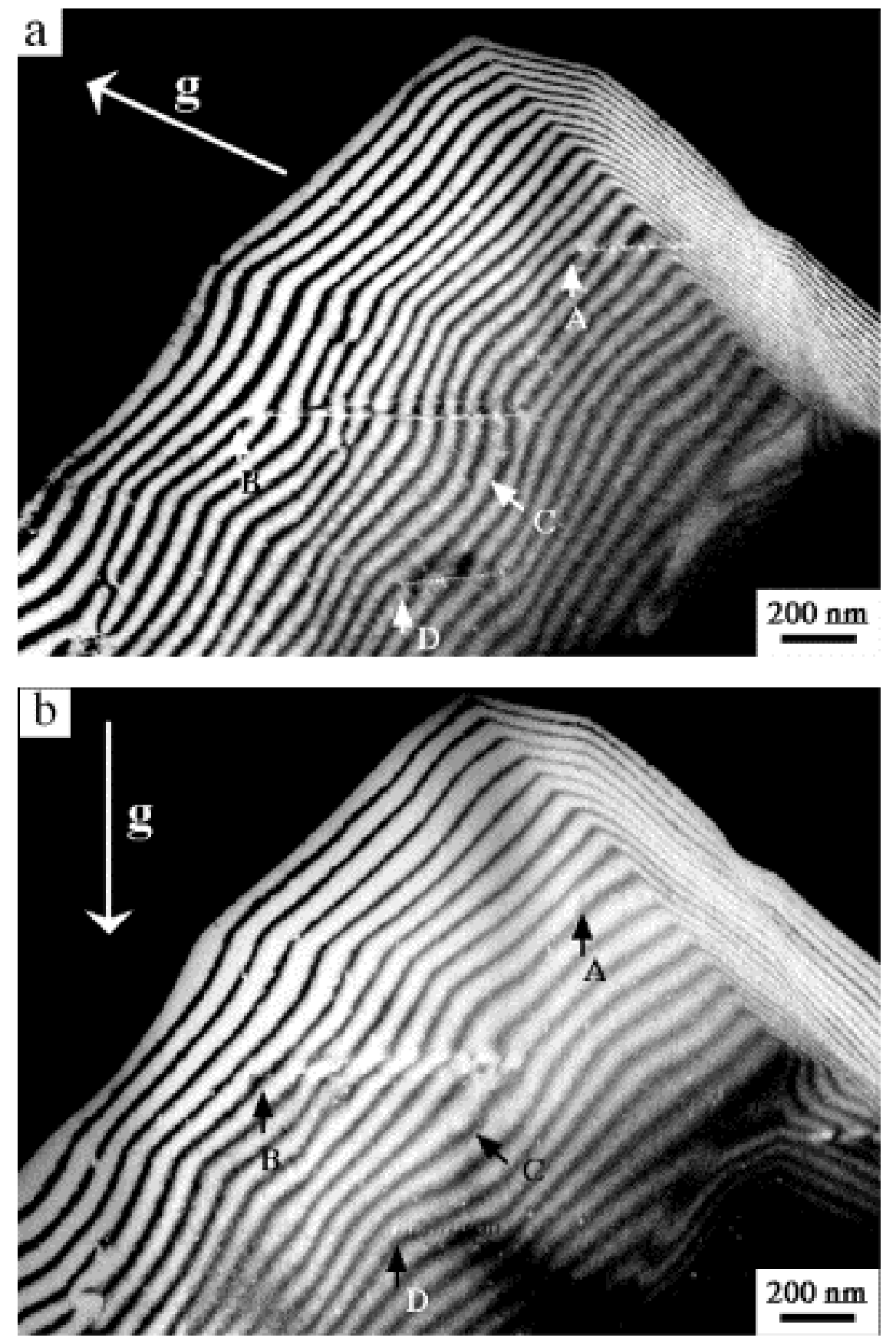

Figure 5 Weak-beam dark-field images with (a) $\mathbf{g} / 5 \mathbf{g}, \mathbf{g}=1101$ and (b) $\mathbf{g} / 5 \mathbf{g}$, $\mathbf{g}=000 \overline{2}$. In (a), the electron beam is nearly parallel to the GaN [11 $\overline{2} 0]$ zone axis, in (b) the GaN [0110] zone axis. 


\section{CONCLUSIONS}

Based on the TEM observation, the following conclusions can be drawn:

- The GaN pyramid, GaN seed layer, and AlN buffer layer in the samples have the following epitactic relationship with respect to the $\mathrm{Si}$ substrate: $[11 \overline{2} 0]_{\mathrm{GaN}}\left\|[11 \overline{2} 0]_{\mathrm{AIN}}\right\|[\overline{1} 10]_{\mathrm{Si}}$ and $(0001)_{\mathrm{GaN}}\left\|(0001)_{\mathrm{AIN}}\right\|(111)_{\mathrm{Si}}$.

- In the core of the pyramid (at or above the seed windows), dislocations thread through the pyramid perpendicular to the interface plane with very high density. Some of these threading dislocations, which originated from the GaN/AlN seed layer, were found to form $90^{\circ}$ bends and half loops at the edge of the pyramid core. The Burgers vector of these half loops is of [0001] type.

- At the lateral growth part of the GaN pyramid, the dislocation density was relatively low. The majority of dislocations thread through the pyramid parallel to the interface plane. The Burgers vectors of most dislocations are $1 / 3\langle 1120\rangle$, they could be either pure screw or mixed dislocations with a $30^{\circ}$ or $60^{\circ}$ angle between the Burgers vector and the dislocation line. Dislocation half loops with Burgers vector $1 / 3<11 \overline{2} 0\rangle$ was also observed. All these defects lie on the (0001) plane.

- Planar defects mostly parallel to the interface plane were observed near the interface.

- The defect density decreases with the distance away from the interface, the top several microns of material being completely defect free.

\section{ACKNOWLEDGMENTS}

This research has been supported by AFOSR (under contract AF/F 48620-95-1-0360), Supplementary support was provided by Honeywell Technology Center and NSF under grant DMR-9522253. The microscopes are part of the CIE Characterization Facility

\section{REFERENCE}

1 H. Asai, S. Adachi, S. Ando, K. Oe, J. Appl. Phys., 55, 3868 (1984)

2 T. Fukai, S. Ando, Y. K. Fukai, Appl. Phys. Lett., 57, 1209 (1990)

3 T. S. Zheleva, O-H. Nam, M. D. Bremser, R. F. Davis, Appl. Phys. Lett., 71, 2472 (1997)

4 Z. Mao, S. McKernan, C. B. Carter, W. Yang, S. A. McPherson, submitted, (1998)

5 Y. Ishida, H. Ishida, K. Kohra, H. Ichinose, Phil. Mag. A, 42, 453 (1980)

6 D. B. Williams, C. B. Carter, Transmission Electron Microscopy-A Textbook for Materials Science, Plenum Press, New York, (1996) 